\title{
Short-Term Electrical Distribution Systems Planning Considering Distributed Generation and Reliability
}

\author{
Rodrigo Rodrigues da Cunha Paiva ${ }^{1} \cdot$ Augusto C. Rueda-Medina $^{2}($ ) \\ José Roberto S. Mantovani ${ }^{2}$
}

Received: 3 October 2016 / Revised: 22 March 2017 / Accepted: 4 May 2017 / Published online: 31 May 2017

(C) Brazilian Society for Automatics-SBA 2017

\begin{abstract}
The distribution systems planning consists of finding an optimized configuration, at a low cost, which allows to keep the quality and reliability of energy supply. Distributed generators (DGs) make the planning problem more complex to be solved. Therefore, it is important to develop efficient computational tools to reduce costs and time in decision making, indicating where, when, and what types of components should be installed and/or replaced in the medium voltage electric distribution system in the presence of DGs. This paper proposes a short-term planning methodology for allocation of DGs, capacitor banks, control and protection devices, as well as the possibility of reconductoring of branches for the distribution network, while maintaining the quality of energy supply. The formulated multi-objective problem consists of minimizing the costs associated with investment, technical losses, and non-supplied energy of the distribution system, subject to physical, economic, and operational constraints. The reliability of the system is improved through allocation of control and protection devices and compact aerial structures. This problem is solved through an approach based on the nondominated sorting genetic algorithm (NSGA-II). Tests were performed in a 135-bus distribution system, and the obtained results show the effectiveness of the proposed methodology.
\end{abstract}

Keywords Distribution systems · Multi-objective optimization $\cdot$ Distributed generation $\cdot$ Reliability

Augusto C. Rueda-Medina

aucerume@gmail.com

1 IFMT - Campus de Cuiabá, Cuiabá, MT, Brazil

2 FEIS UNESP, Ilha Solteira, SP, Brazil

\section{List of symbols}

The notation used throughout this paper is reproduced below for quick reference.

Sets:

$\begin{array}{ll}\Omega_{\mathrm{D}} & \text { Load demand levels. } \\ \Omega_{\mathrm{Br}} & \text { Branches of the system. } \\ \Omega_{\mathrm{Bus}} & \text { Buses of the system. } \\ \Omega_{\mathrm{Cbl}} & \begin{array}{l}\text { Types of cables mounted in conven- } \\ \text { tional aerial, compact, and isolated } \\ \end{array} \\ & \begin{array}{l}\text { structures. } \\ \text { Types of capacitor banks (fixed and }\end{array} \\ \Omega_{\mathrm{CB}} & \text { switched), as well as their corre- } \\ & \text { sponding nominal reactive power. } \\ \Omega_{\mathrm{Prt}} & \text { Protection devices (switches, fuse } \\ & \text { links, reclosers, and directional } \\ & \text { relays). } \\ \Omega_{\mathrm{DG}} & \text { Distributed generators. } \\ \Omega_{\mathrm{SBus}, s} & \text { Buses of section } s . \\ \Omega_{\mathrm{Sc}} & \text { Probabilistic scenarios. }\end{array}$

Constants:

$c_{\mathrm{Jou}, d}$

$c_{\mathrm{NSE}, d}$

$\rho_{d}$

$R_{i j}, G_{i j}, B_{i j}$

$c_{\mathrm{Cbl}, k}$

$L_{i j}$

$c_{\mathrm{CB}, k}$
Costs of the Joule effect losses in load level $d$.

Costs of the non-supplied energy in load level $d$.

Number of hours in a year in load level $d$.

Resistance, conductance, and susceptance, respectively, of branch $i j$. Cost of a $k$-type cable.

Length of cable in branch $i j$. Cost of a $k$-type capacitor bank. 


$$
\begin{aligned}
& c_{\mathrm{Prt}, k} \\
& P_{\mathrm{D}, i, d}, Q_{\mathrm{D}, i, d} \\
& P_{\mathrm{DG}, g, d}^{\mathrm{Min}}, Q_{\mathrm{DG}, g, d}^{\mathrm{Min}} \\
& P_{\mathrm{DG}, g, d}^{\mathrm{Max}}, Q_{\mathrm{DG}, g, d}^{\mathrm{Max}} \\
& p f_{\mathrm{DG}}^{\mathrm{Min}}, p f_{\mathrm{DG}}^{\mathrm{Max}} \\
& V^{\mathrm{Min}}, V^{\mathrm{Max}} \\
& I_{i}^{\mathrm{Max}}
\end{aligned}
$$$$
n_{\mathrm{DG}}, n_{\mathrm{CB}}, n_{\mathrm{FL}}
$$$$
n_{\mathrm{Sw}}, n_{\mathrm{Rc}}, n_{\mathrm{DR}}
$$$$
n_{\mathrm{RcDR}}^{\mathrm{Max}}
$$$$
n_{\mathrm{SPrt}}^{\mathrm{Max}}
$$$$
n_{\mathrm{S}}, n_{\mathrm{D}}
$$$$
\Gamma_{\mathrm{Rep}}, \Gamma_{\mathrm{Res}}
$$$$
L_{S}
$$$$
\lambda_{\mathrm{F}, s}
$$$$
\lambda_{\mathrm{St}, s}
$$

$\eta_{\text {Rep }, f, s} \eta_{\text {Res, } f, s}$

$N$

$p_{g, i, j}$

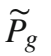

$n_{\mathrm{Sc}}$

$n_{\mathrm{Yr}}$

$\alpha$
Cost of protection device $k$.

Active and reactive power demanded in bus $i$ and load level $d$. Minimum limits of active and reactive power generation of distributed generator $g$ in load level $d$.

Maximum limits of active and reactive power generation of distributed generator $g$ in load level $d$.

Minimum and maximum limits of the distributed generators power factor.

Minimum and maximum voltage magnitude limits.

Maximum current limit in branch ij.

Number of available distributed generators, capacitor banks, and fuse links, respectively.

Number of available switches, reclosers, and directional relays, respectively.

Maximum number of available reclosers and directional relays. Maximum number of protection devices connected in series.

Number of feeder sections and load levels, respectively.

Repair and restoration times, respectively.

Length of section $s$.

Fault index in a year in section $s$.

Index that represents the influence of the structure in a fault in section $s$.

Binary constant associated with the influence or not of the repair and restoration of a fault $f$ in section $s$, respectively.

Size of population in the multiobjective genetic algorithm.

Transition probability of distributed generator $g$ of moving from an initial generation state $i$ to a final generation state $j$.

Matrix of transition probabilities of distributed generator $g$.

Number of probabilistic scenarios. Number of years of the planning horizon.

Investment return rate.
Variables:

$C_{\text {Tech }}$
$C_{\text {Inv }}$
$I_{i j, d}$
$V_{i, d}$
$\theta_{i j, d}$
$E_{\mathrm{NS}, d}$

$\eta_{\mathrm{DG}, i}, \eta_{\mathrm{CB}, i}, \eta_{\mathrm{Prt}, i}$

$\eta_{\mathrm{Cbl}, i j}$

$\eta_{\mathrm{Sw}, i}, \eta_{\mathrm{Rc}, i}, \eta_{\mathrm{DR}, i}$

$P_{\mathrm{DG}, g, i, d}, Q_{\mathrm{DG}, g, i, d}$

$P_{i, d}, Q_{i, d}$

$Q_{\mathrm{CB}, k, i, d}$

$p f_{g, i, d}$

FV

$P V$

\section{Introduction}

The development of models and techniques for solving the distribution systems' operating and expansion planning problem aims at finding an optimal configuration at a low investment costs (reform or expansion). Operating and investment costs are minimized considering physical and operational constraints of the network in order to meet consumers with quality, reliability, and competitive costs (Gonen 1985).
Costs associated with the network technical losses and non-supplied energy.

Costs of investments in equipment that must be installed.

Current in branch $i j$ in load level $d$. Magnitude of voltage in bus $i$ in load level $d$.

Difference between angles of voltages in buses $i$ and $j$ in load level $d$.

Non-supplied energy in load level $d$.

Binary variables associated, respectively, with the existence or not of a distributed generator, capacitor bank, and/or protection device to be installed in bus $i$.

Binary variable associated with the existence or not of a cable to be installed/replaced in branch $i j$.

Binary variables associated, respectively, with the existence or not of a switch, recloser, and/or directional relay to be installed in bus $i$.

Active and reactive power generated by distributed generator $g$ in bus $i$ and load level $d$, respectively. Active and reactive power injected in bus $i$ and load level $d$, respectively.

Reactive power injected in bus $i$ by capacitor bank $k$ in load level $d$.

Power factor of distributed generator $g$ in bus $i$ and load level $d$.

Future value.

Present value. 
This challenge becomes even more complex when there are installed distributed generators (DG) in the network. The energy generation from wind power has been increasing every year in the energy matrix of several countries. The immediate consequence of this new scenario is the increase in the number of requests, to the planning department of electric distribution companies (EDCs), for authorization to connect generators directly into the electrical distribution system (EDS).

The connection of new sources changes some network characteristics, such as voltages, losses, protection scheme, direction of flow, among others. Therefore, the consumers want to ensure the satisfactory of the minimum reliability requirements and the greatest possible availability of energy supply. Thus, for the planning departments of the EDCs, the development of computational tools to reduce costs and time in decision making is important, indicating where, when, and what types of components should be installed and/or replaced in the distribution network in the presence of DGs.

Thus, it is important to have better connection planning of the DGs in the distribution network, as well as a mechanism to minimize conflicts caused by the installation of these sources into the network (e.g., conflicts when the EDC is not the owner of the DGs). Some of these possible conflict points are:

1. To keep the voltage levels and quality of energy supply within the standards established by regulatory agencies.

2. To keep the technical losses in the same or lower levels than the existing ones without the connection of DGs.

3. To keep the lowest possible investment in the network to encourage the connection of DGs (owned by the EDC or independent producers) without deteriorating the quality of energy supply.

In the short-term planning of distribution systems, some devices and actions allow effective control over voltage, active and reactive power, power factor, among others, which can be used to keep the quality and reliability of energy supply. Among these devices and actions, there are the allocation of capacitor banks (CBs), reconductoring of branches of the distribution network, and allocation of control and protection devices.

The DGs are part of the planning problem as additional elements that could be considered to meet economic interests of EDCs and independent energy producers. The DGs must be installed in appropriate places, according to the availability of primary energy sources, in order to bring the maximum benefits to the system, such as losses reduction, voltage control, relief in the conductors current flow, improved quality of energy supply, among others (El-kathan et al. 2005). Besides the technical benefits, another major benefit that the DGs can offer is related to the environment, since the progress and reduction of costs of control devices, which use power electronics technology, and the improvement of communications systems allows the use of renewable energy sources in medium and low voltage networks, i.e., the use of clean or inexhaustible energy sources is increasingly encouraged (Tan et al. (2013)).

In recent years, many studies involving short-term action planning were developed; however, in most of them, the problems of CBs allocation, voltage regulators, and reconductoring of branches of the distribution network are treated separately. Regarding the CBs, noteworthy is the work presented in Baran and Wu (1989), in which this problem is formulated as a mixed-integer nonlinear programming (MINLP) problem. The authors propose the approximation of the non-differentiable objective function to a linear objective function, and they solve the problem using Benders' decomposition with continuous variables. Thereafter, classical optimization techniques, mono- and multi-objective heuristics, and metaheuristics techniques have been proposed for solving the CBs allocation problem (Chiang et al. 1990; Sundharajan and Pahwa 1994; Gallego et al. 2001; Souza et al. 2004; Park et al. 2009). Reconductoring of branches of the feeder of the distribution network is another action of the short-term planning problem. Several models and techniques for solving this problem can be found Mandal and Pahwa (2002), Mendoza et al. (2006), Vahidet et al. (2009). There are also works which propose models and solution techniques for solving simultaneously the CBs allocation and reconductoring problems (Pereira et al. 2013).

In this paper, different from the works presented in the literature, it is proposed a methodology for simultaneous allocation of DGs, fixed and switched CBs, control and protection devices, as well as the possibility of reconductoring of branches of the feeder of the distribution network with possible changes of the cables support structures. This problem is formulated as a multi-objective MINLP problem, which is difficult to solve due to its combinatorial nature, presenting a large search space, as well as a multimodal structure with large number of local optimal solutions. Thereby, the main contribution of this work is to bring together several elements of the planning and operation problem in a single model, making it closer to reality. The DGs are represented deterministically and also through modeling uncertainties considering the wind incidence probability in the wind power generation units that can be connected on the network. The reliability of the network is evaluated considering the cost of the non-supplied energy. Improvement in the network reliability is obtained considering that the proposed mathematical model has the ability to decide, according to technical and economic aspects, the change of conventional aerial structures by simple or isolated compact structures, and also through the optimized allocation of control and protection devices. The non-dominated sorting genetic algorithm 
(NSGA-II) (Deb et al. (2002)) is used for the solution of the proposed problem. To validate the proposed methodology, the results of tests carried out on a 135-bus distribution system (LaPSEE, http://www.feis.unesp.br/\#!/departamentos/ engenharia-eletrica/pesquisas-e-projetos/lapsee/english/do wnloads/testing-systems/) are presented.

This paper is organized as follows: In Sect. 2, the assumptions and mathematical formulation proposed in this work are presented; in Sect. 3, the NSGA-II implemented for solving the problem under study is described; in Sect. 4, the approach to address the uncertainties of wind power generation units is presented; in Sect. 5, the results of performed tests and corresponding analysis are shown; and, finally, the conclusions are exposed in Sect. 6.

\section{Assumptions and Mathematical Formulation}

The assumptions and mathematical formulation of the proposed short-term electrical distribution system planning (EDSP) are presented in the next subsections.

\subsection{Assumptions}

In order to present the mathematical formulation of the proposed EDSP problem, some assumptions regarding the properties and physical behavior of the equipment and devices that could be allocated in the distribution network were made. These assumptions are as follows:

1. The DGs may be owned either by the EDC or by independent energy producers.

2. DGs with a high degree of variability are considered in this work. To consider these kinds of DGs as dispatchable, their variability is reduced by using a forecasting system, which combines the Monte Carlo method (MCM) and Markov models (MkvM) (Rueda-Medina and PadilhaFeltrin 2013).

3. The DGs have anti-islanding protection, and their islanded operation is not allowed.

4. In the construction of new distribution circuits or reconductoring of the existing ones, it is contemplated that new lines can be built or conventional aerial structures can be replaced with compact or isolated structures, which have higher reliability indices.

5. Fixed and switched capacitor banks can be allocated.

6. Sections are defined as the network segments which can be isolated by switching actions in control and protection devices.

7. In the model, it is considered the distribution network restoration through optimal allocation of sectionalizing switches when there are permanent faults in each of the predefined sections of the feeder.
8. It is considered that protection devices operate in a selective and coordinated manner, so that improper actions of fuses with respect to temporary disturbances are reduced through the action of reclosers.

9. In contingencies, the protection devices must act to protect the system, and control devices must be switched (open or close) to isolate the area under disturbance, as well as to transfer interrupted loads to neighboring feeders.

\subsection{Mathematical Formulation}

The proposed short-term EDSP problem, considering economic analysis of the distributed generation allocation and improvements in the network reliability, can be mathematically formulated as a multi-objective MINLP problem, as presented as follows.

$$
\begin{aligned}
\operatorname{Min} C_{\mathrm{Tech}}=\sum_{d \in \Omega_{\mathrm{D}}} \sum_{i j \in \Omega_{\mathrm{Br}}} \rho_{d} c_{\mathrm{Jou}, d} R_{i j} I_{i j, d}^{2} \\
\quad+\sum_{d \in \Omega_{\mathrm{D}}} \rho_{d} c_{\mathrm{NSE}, d} E_{\mathrm{NS}, d}
\end{aligned}
$$

Min $C_{\mathrm{Inv}}=\sum_{k \in \Omega_{\mathrm{Cb} 1} i} \sum_{j \in \Omega_{\mathrm{Br}}} c_{\mathrm{Cbl}, k} \eta_{\mathrm{Cbl}, i j} L_{i j}$

$$
+\sum_{k \in \Omega_{\mathrm{CB}} i \in \Omega_{\mathrm{Bus}}} \sum_{\mathrm{CB}, k} \eta_{\mathrm{CB}, i}+\sum_{k \in \Omega_{\mathrm{Pr} t}} \sum_{\in \Omega_{\mathrm{Bus}}} c_{\mathrm{Prt}, k} \eta_{\mathrm{Prt}, i}
$$

Subject to:

$$
\begin{aligned}
& \sum_{g \in \Omega_{\mathrm{DG}}} P_{\mathrm{DG}, g, i, d}-P_{\mathrm{D}, i, d} \\
& -V_{i, d} \sum_{j \in \Omega_{\mathrm{Bus}} ; j \neq i} V_{j, d}\left(G_{i j} \cos \theta_{i j}+B_{i j} \sin \theta_{i j}\right)=0 ; \\
& \forall i \in \Omega_{\mathrm{Bus}} ; \forall d \in \Omega_{\mathrm{D}}
\end{aligned}
$$

$$
\begin{aligned}
& \sum_{g \in \Omega_{\mathrm{DG}}} Q_{\mathrm{DG}, g, i, d}+Q_{\mathrm{CB}, k, i, d}-Q_{\mathrm{D}, i, d} \\
& -V_{i, d} \sum_{j \in \Omega_{\mathrm{Bus}} ; j \neq i} V_{j, d}\left(G_{i j} \sin \theta_{i j}-B_{i j} \cos \theta_{i j}\right)=0 ; \\
& \forall i \in \Omega_{\mathrm{Bus}} ; \forall k \in \Omega_{\mathrm{CB}} ; \forall d \in \Omega_{\mathrm{D}} \\
& P_{\mathrm{DG}, g, d}^{\mathrm{Min}} \leq P_{\mathrm{DG}, g, i, d} \leq P_{\mathrm{DG}, g, d}^{\mathrm{Max}} \\
& \forall g \in \Omega_{\mathrm{DG}} ; \forall i \in \Omega_{\mathrm{Bus}} ; \forall d \in \Omega_{\mathrm{D}} \\
& Q_{\mathrm{DG}, g, d}^{\mathrm{Min}} \leq Q_{\mathrm{DG}, g, i, d} \leq Q_{\mathrm{DG}, g, d}^{\mathrm{Max}} \\
& \forall g \in \Omega_{\mathrm{DG}} ; \forall i \in \Omega_{\mathrm{Bus}} ; \forall d \in \Omega_{\mathrm{D}}
\end{aligned}
$$




$$
\begin{aligned}
& \begin{array}{l}
p f_{\mathrm{DG}}^{\mathrm{Min}} \leq p f_{g, i, d} \leq p f_{\mathrm{DG}}^{\mathrm{Max}} \\
\forall g \in \Omega_{\mathrm{DG}}
\end{array} ; i \in \Omega_{\mathrm{Bus}} ; \forall d \in \Omega_{\mathrm{D}} \\
& V^{\mathrm{Min}} \leq V_{i, d} \leq V^{\mathrm{Max}} ; \quad \forall i \in \Omega_{\mathrm{Bus}} ; \forall d \in \Omega_{\mathrm{D}} \\
& I_{i j, d} \leq I_{i}^{\mathrm{Max}} ; \\
& \sum_{i \in \Omega_{\mathrm{Bus}}} \eta_{\mathrm{DG}, i} \leq n_{\mathrm{DG}} \quad \forall i j \in \Omega_{\mathrm{Br}} \\
& \sum_{i \in \Omega_{\mathrm{Bus}}} \eta_{\mathrm{CB}, i} \leq n_{\mathrm{CB}} \\
& \sum_{i \in \Omega_{\mathrm{Bus}}} \eta_{\mathrm{FL}, i} \leq n_{\mathrm{FL}} \\
& \sum_{i \in \Omega_{\mathrm{Bus}}} \eta_{\mathrm{Sw}, i} \leq n_{\mathrm{Sw}} \\
& \sum_{i \in \Omega_{\mathrm{Bus}}} \eta_{\mathrm{Rc}, i}+\sum_{i \in \Omega_{\mathrm{Bus}}} \eta_{\mathrm{DR}, i} \leq n_{\mathrm{RcDR}}^{\mathrm{Max}}, i \leq n_{\mathrm{SPrt}}^{\mathrm{Max}} \\
& \sum_{\mathrm{Bus}}
\end{aligned}
$$

In proposed model (1)-(15), two objective functions are considered. The first objective function, expression (1), represents an improvement measure of the network reliability, and it corresponds to the minimization of the costs associated with the network technical losses and non-supplied energy; the second objective function, expression (2), corresponds to the minimization of the costs for investments in the equipment that must be installed to meet reliability and quality of energy supply. The non-supplied energy in the second term in (1) is obtained according to expression (16). In (2), the first term corresponds to the costs associated with the cable structure (conventional aerial, compact, or isolated) which can be installed/replaced in the respective network section; the second term corresponds to the installation costs of $\mathrm{CBs}$, according to their characteristics (fixed or switched) and power ratings; and the third term corresponds to the costs of control and protection devices, such as switches, fuse links, reclosers, and directional relays, which can be installed in the network.

$$
\begin{aligned}
E_{\mathrm{NS}, d}= & \Gamma_{\mathrm{Rep}} \sum_{f \in \Omega_{\mathrm{F}} \in \Omega_{\mathrm{S}}} \sum_{s} L_{s} \lambda_{\mathrm{F}, s} \lambda_{\mathrm{St}, s} \eta_{\mathrm{Rep}, f, s} \sum_{i \in \Omega_{\mathrm{SBus}, \mathrm{s}}} P_{\mathrm{D}, i, d} \\
& +\Gamma_{\operatorname{Res}} \sum_{f \in \Omega_{\mathrm{F}} \in \Omega_{\mathrm{S}}} \sum_{s} L_{\mathrm{F}, s} \lambda_{\mathrm{St}, s} \eta_{\mathrm{Res}, f, s} \sum_{i \in \Omega_{\mathrm{SBus}, \mathrm{s}}} P_{\mathrm{D}, i, d}
\end{aligned}
$$

Constraints (3) and (4) are the conventional representation of the active and reactive power flow balance, respectively. Active and reactive power generation of the DGs must be within operational limits, according to (5) and (6), respectively. The power factor of the DGs, both leading and lagging, must be within specified limits, as represented in (7). Voltages in buses must be within the specified limits, as presented in (8), and currents in branches must be less than the specified maximum values, as shown in (9). The maximum number of DGs, CBs, fuse links, switches, reclosers, and directional relays that can be installed in the system is represented by constraints (10)-(14), respectively; note that, when a DG is installed between a recloser and the substation, such recloser has to be replaced by a directional relay. Finally, the number of protection devices connected in series must be less than a specified maximum number, as represented in (15).

\section{Solution Techniques}

The proposed short-term EDSP problem is a MINLP, NPhard, that is difficult to be solved through exact classical optimization techniques. Among the techniques reported in the literature to solve this kind of problem, the NSGA-II (Deb et al. 2002) is used in this work.

The NSGA-II, widely reported in the literature, e.g., in Pereira et al. (2013) and Padilha-Feltrin et al. (2015), is an elitist algorithm that preserves the diversity of the population. This algorithm is robust, easy to understand and to implement and can be applied to several kinds of problems with excellent results and low computational times. The NSGA-II incorporates the domination principle to find a set of non-dominated high-quality solutions, which may be near or even belong to the Pareto-optimal front. The flowchart of the NSGA-II implemented to the proposed problem is presented in Fig. 1, and its main features are explained in the next subsections.

The power flow is an essential tool to determine the fitness function values in the NSGA-II implemented to solve the problem presented in this work. Bearing in mind factors such as speed of convergence, accuracy, and robustness, a power flow based on the backward-forward method was used for this proposal (Shirmohammadi et al. 1988).

\subsection{Individuals Coding}

The coding scheme used to represent the individuals or chromosomes in the NSGA-II consists of decimal and binary numbers, which are grouped in four main sets to represent the types of cables/structures, DGs, CBs, and the types of protection equipment that should be installed in the network, as detailed in Fig. 2.

Set 1, shown in Fig. 2, which corresponds to the reconductoring cable/structure (conventional aerial, compact, and 


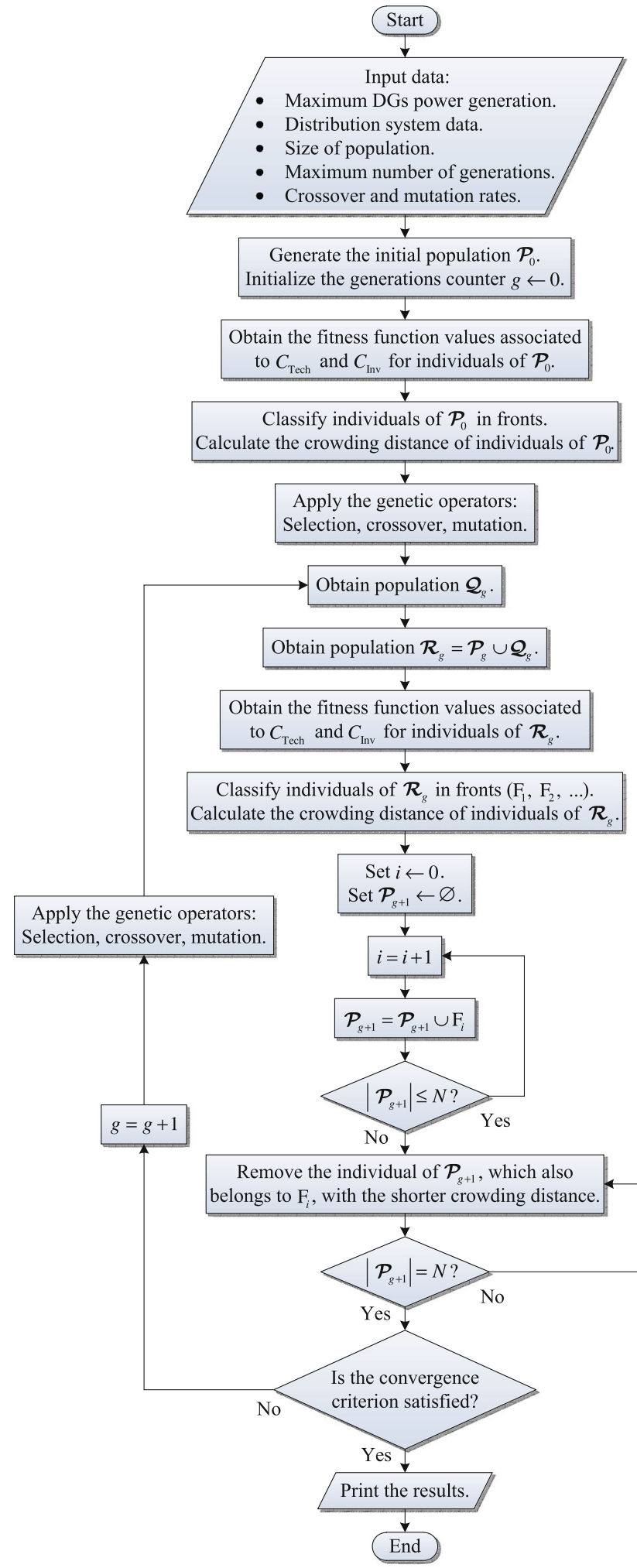

Fig. 1 Flowchart of the NSGA-II implemented to solve the proposed problem

isolated), is composed of $n_{\mathrm{S}}$ fields or genes associated with the number of sections in which the feeder is previously divided. Each field of this set contains an integer

\begin{tabular}{|c|c|c|c|c|c|c|c|c|c|c|}
\hline \multirow{3}{*}{\multicolumn{2}{|c|}{$\begin{array}{c}\text { Set } 1 \\
\text { (Cables/ } \\
\text { Structures) }\end{array}$}} & \multicolumn{9}{|c|}{ Set 2 (DGs) } \\
\hline & & \multirow{2}{*}{\multicolumn{2}{|c|}{$\begin{array}{l}\text { Allocation } \\
\text { buses }\end{array}$}} & \multicolumn{7}{|c|}{ Power factor } \\
\hline & & & & $\mathrm{DG}_{1}$ & \begin{tabular}{l|l}
1 & $\cdots$
\end{tabular} & $\mathrm{DG}_{1}$ & \begin{tabular}{|l|}
$\ldots$ \\
\end{tabular} & $\mathrm{DG}_{n_{\mathrm{DG}}}$ & $\ldots$ & \multirow{2}{*}{\begin{tabular}{|c|}
$\mathrm{DG}_{n_{\mathrm{DG}}}$ \\
$p f_{n_{\mathrm{DG}}, \mathrm{GB}_{n_{\mathrm{OG}}}, n_{\mathrm{D}}}$
\end{tabular}} \\
\hline $\mathrm{CS}$ & \begin{tabular}{|l|l|l|}
$\Gamma_{1}$ & $\ldots$ & $\mathrm{CST}_{n_{\mathrm{s}}}$ \\
\end{tabular} & $\mathrm{GB}_{1}$. & \begin{tabular}{l|l}
.. & $\mathrm{GB}_{n_{\mathrm{DC}}}$ \\
\end{tabular} & $p f_{1, \mathrm{~GB}}$ & \begin{tabular}{l|l}
$\beta_{1}, 1$ & $\cdots$ \\
\end{tabular} & $p f_{1, \mathrm{~GB}_{1}, n}$ & $\begin{array}{ll}y_{1} & \cdots \\
\end{array}$ & $p f_{n_{\mathrm{DG}}, \mathrm{GB}_{m_{\mathrm{j}}},}$ & $\begin{array}{ll}1 . \cdots \\
\end{array}$ & \\
\hline \multicolumn{11}{|c|}{ Set 3 (CBs) } \\
\hline \multirow{3}{*}{.. } & \multirow{2}{*}{\multicolumn{2}{|c|}{$\begin{array}{l}\text { Allocation } \\
\text { buses }\end{array}$}} & \multicolumn{8}{|c|}{ Reactive power } \\
\hline & & & $\mathrm{CB}_{1}$ & ... & & $\mathrm{CB}_{1}$ & $\ldots$ & $\mathrm{CB}_{n_{\mathrm{CB}}}$ & $\ldots$ & $\mathrm{CB}_{n_{\mathrm{CB}}}$ \\
\hline & \begin{tabular}{l|l|l}
$\mathrm{CBB}_{1}$ & $\ldots$ & $\mathrm{C}$ \\
\end{tabular} & $\mathrm{BB}_{n_{\mathrm{CB}}}$ & $Q_{\mathrm{CB}, 1, \mathrm{CB}}$ & $\ldots$ & $Q_{\mathrm{CB}}$ & $\mathrm{BB}_{1}, n_{\mathrm{p}}$ & $\ldots \mid Q$ & $\mathrm{CB}, n_{\mathrm{CB}}, \mathrm{CBB}_{n_{\mathrm{Ca}}}$, & $\cdots$ & $Q_{\mathrm{CB}, n_{\mathrm{CB}}, \mathrm{CBB}_{n_{\mathrm{Ca}},}, n_{\mathrm{D}}}$ \\
\hline \multicolumn{11}{|c|}{ Set 4 (Protection equipment) } \\
\hline \multirow[t]{2}{*}{$\cdots$} & \multicolumn{2}{|c|}{ Fuse links } & \multicolumn{3}{|c|}{ Switches } & \multicolumn{2}{|c|}{ Reclosers } & \multicolumn{3}{|c|}{$\begin{array}{l}\text { Directional } \\
\text { relays }\end{array}$} \\
\hline & \begin{tabular}{l|l|l}
$\mathrm{BFL}_{1}$ & $\ldots$ & $\mathrm{B}$ \\
\end{tabular} & $\mathrm{L}_{n_{\mathrm{rL}}}$ & $\mathrm{BSW}_{1}$. & BSV & & \begin{tabular}{l|l|}
$\mathrm{RC}_{1}$ & $\cdots$ \\
\end{tabular} & BRC & $\mathrm{C}_{n_{\mathrm{Rc}}} \mid \mathrm{BDR}_{1}$ & $\ldots \mathrm{B}$ & $\mathrm{R}_{n_{\mathrm{DR}}}$ \\
\hline
\end{tabular}

Fig. 2 Coding scheme of each individual in the NSGA-II

number, $\mathrm{CST}_{1}$ to $\mathrm{CST}_{n_{\mathrm{S}}}$, representing one of the available cable/structure types.

The buses where the DGs could be allocated, i.e., $\mathrm{GB}_{1}$ to $\mathrm{GB}_{n_{\mathrm{DG}}}$, and the power factor of the DGs for all load levels, i.e., $p f_{1, \mathrm{~GB}_{1}, 1}$ to $p f_{n_{\mathrm{DG}}, \mathrm{GB}_{n_{\mathrm{DG}}}, n_{\mathrm{D}}}$ are specified in Set 2 , as presented in Fig. 2.

Regarding the $\mathrm{CBs}$, the candidate allocation buses, i.e., $\mathrm{CBB}_{1}$ to $\mathrm{CBB}_{n_{\mathrm{CB}}}$ and the corresponding reactive power for all load levels, i.e., $Q_{\mathrm{CB}, 1, \mathrm{CBB}_{1}, 1}$ to $Q_{\mathrm{CB}, n_{\mathrm{CB}}, \mathrm{CBB}_{n_{\mathrm{CB}}}, n_{\mathrm{D}}}$ are represented in Set 3, as shown in Fig. 2.

Set 4 contains information regarding the allocation branches of fuse links, switches, reclosers, and directional relays, i.e., $\mathrm{BFL}_{1}$ to $\mathrm{BFL}_{n_{\mathrm{FL}}}, \mathrm{BSW}_{1}$ to $\mathrm{SWL}_{n_{\mathrm{Sw}}}, \mathrm{BRC}_{1}$ to $\mathrm{BRC}_{n_{\mathrm{Rc}}}$, and $\mathrm{BDR}_{1}$ to $\mathrm{BDR}_{n_{\mathrm{DR}}}$, respectively. A zero value in any field of this set means that there is not a protection device installed in the corresponding branch.

\subsection{Initialization, Sorting, and Crowding Distance}

According to the features of the problem under study, there are several methods to generate the initial population for the multi-objective algorithm. Usually, the population is generated in a random or pseudorandom basis. In this work, the initial population $\mathcal{P}_{0}$, with size $N$, is generated in a pseudorandom basis using the nominal values and minimum and maximum capacities of cables/structures, DGs, CBs, and protection devices.

In each generation of the NSGA-II, the population $\mathcal{P}$ is sorted according to the non-dominated individual levels. All individuals are classified in fronts of non-domination $F_{1}, F_{2}$, $\ldots, \mathrm{F}_{i}$ (the sorting is done considering that, as the smaller is the rank of the front, better is the solution).

The sorting is performed using the restricted domination operator, defined in Deb et al. (2002), as follows:

- Every feasible solution $x$ dominates all unfeasible solutions $y$. 
Table 1 Costs of cables/structures

\begin{tabular}{|c|c|c|c|c|c|c|c|c|c|c|c|}
\hline \multirow[t]{2}{*}{ Cable/structure type } & \multicolumn{5}{|c|}{ Conventional aerial } & \multicolumn{3}{|c|}{ Compact } & \multicolumn{3}{|l|}{ Isolated } \\
\hline & 1 & 2 & 3 & 4 & 5 & 6 & 7 & 8 & 9 & 10 & 11 \\
\hline Cost (US\$/km) & 1539 & 3077 & 4616 & 6154 & 7693 & 11,947 & 13,870 & 15,794 & 21,154 & 23,077 & 25,000 \\
\hline
\end{tabular}

- If $x$ and $y$ are both feasible solutions, $x$ dominates $y$ only if $x$ is better than $y$ at least in one objective function, and $x$ is not worse than $y$ in no other objective function.

- If $x$ and $y$ are both infeasible solutions, $x$ dominates $y$ only if $x$ is less infeasible than $y$ at least in one problem constraint, and $x$ is not worse than $y$ in no other problem constraint.

For each individual, a crowding distance is also calculated. This distance is an estimation of the size of the search space around the solution under analysis that is not occupied by another solution (Deb et al. 2002).

\subsection{Genetic Operators}

The genetic operators called tournament selection, crossover, and mutation are applied to obtain a descendant population $\mathcal{Q}$ with size $N$ (Deb 2009).

\subsubsection{Tournament Selection}

The criteria used in the tournament selection are based on the crowding comparison operator (Deb et al. 2002): If two solutions belong to the same front, the solution with the greatest crowding distance is selected, in order to preserve the diversity of the population; otherwise, the solution which belongs to the front with the best domination level is selected.

\subsubsection{Crossover}

In this work, the crossover is performed in multiple points of a pair of selected individuals (parents) from the population $\mathcal{P}$. Random locations are selected in each of the parental individual in order to divide it in several parts. Each descendant comprises the combination of these parts, such that the genetic information of both parents is passed to the descendants.

After applying the crossover operator, a new population $\mathcal{Q}$ is generated. A set containing the combination of parents and descendant population $\mathcal{R}=\mathcal{P} \cup \mathcal{Q}$ is classified into fronts, according to the non-domination levels, as explained in Sect. 3.2.

A new population is then generated using the individuals of $\mathcal{R}$. The new population generation process consists of adding individuals of $\mathcal{R}$ to a new population set, beginning with the individuals which belong to the best fronts. This process is repeated until the number of individuals in the new population set exceeds $N$. Next, the individuals with the shorter crowding distances of the new population set, which also belong to the last front added to this set, are removed until the number of individuals $N$ is reached.

\subsubsection{Mutation}

The mutation of individuals is used to ensure greater scanning in the search space, avoiding local optimal solutions. In this work, the mutation process consists of changing the value of a random location of a selected individual. The decision whether or not an individual changes depends on a predefined probability mutation rate.

The process to perform the mutation operator to each of the sets of the individuals coding (see Fig. 2) is presented below:

- Set 1 (cables/structures): In this set, in a randomselected location, the mutation is performed changing the cable/structure type from a predefined list (Table 1).

- Set 2 (DGs): As presented in Fig. 2, this set is divided into 2 subsets, which correspond to the allocation bus and power factor of the DGs. If the random-selected location of the mutation is in the allocation bus subset, a new bus value, chosen randomly from a candidate allocation buses set, replaces the existing bus value. Otherwise, if the mutation is in the power factor subset, new values (for all load levels) are chosen randomly between the predefined minimum and maximum operational power factor limits.

- Set 3 (CBs): Similar to Set 2, this set is divided into 2 subsets, which correspond to the allocation bus and to the reactive power value of the CBs. In this set, the mutation is performed as follows:

- Initially, a random-selected location in the allocation buses subset is defined. The actual value associated with this location is then changed from 0 to 1 or 1 to 0 .

- If the new value is 0 , then all values associated with the corresponding $\mathrm{CB}$ in the reactive power subsets (for all load levels) are also changed to 0 . 
Table 2 Costs of acquisition, installation, and maintenance of protection equipment

\begin{tabular}{lllllll}
\hline Fuse link & Current (A) & $0 \leq 20$ & $21 \leq 50$ & $51 \leq 80$ & $81 \leq 100$ & $101 \leq 200$ \\
& Cost (US\$) & 500 & 800 & 950 & 1100 & 1200 \\
Recloser & Current (A) & $0 \leq 50$ & $51 \leq 100$ & $101 \leq 300$ & $301 \leq 500$ & $501 \leq 1000$ \\
& Cost (US\$) & 16,000 & 20,000 & 23,000 & 28,000 & 31,000 \\
Directional relay & Current (A) & $0 \leq 50$ & $51 \leq 100$ & $101 \leq 200$ & $201 \leq 500$ & $501 \leq 1,000$ \\
& Cost (US\$) & 7000 & 7500 & 8000 & 8500 & 9000 \\
\hline
\end{tabular}

- If the new value is 1 , then new values are chosen randomly between the predefined minimum and maximum operational limits of the corresponding $\mathrm{CB}$.

- Set 4 (protection equipment): In this set, the mutation process consists of changing the value of a random-selected location, which corresponds to the installation branch of fuse links, switches, reclosers, or directional relays. The new value of the selected location in this subsets is chosen randomly from a predefined options list (Table 2). A zero new value means that there is no switch, fuse link, recloser, or directional relay installed in the corresponding branch.

\section{Distributed Generators Uncertainties}

In this work, an algorithm proposed in Rueda-Medina and Padilha-Feltrin (2013), which combines the MCM and MkvM, is used to reduce the generation uncertainties of the DGs which use wind as the primary energy source. In this algorithm, the time series for the active power outputs of these DGs are defined through the MkvM theory and, using the MCM, a set of probabilistic scenarios is generated. In Fig. 3, the MCM algorithm used in this proposal is shown.

For each DG $g$, a set of discretized generation states (GSs) is defined, and MkvM are described in terms of the transition probabilities of moving between these GSs (e.g., from an initial GS $i$ to a final GS $j$, say probability $p_{g, i, j}$. These probabilities depend on collected statistical data (e.g., wind speed), and they form the matrix of transition probabilities $\widetilde{P}_{g}$ presented in (17).

$$
\begin{aligned}
& \widetilde{P_{g}}=\left[\begin{array}{ccccc}
p_{g, 1,1} & \cdots & p_{g, 1, j} & \cdots & p_{g, 1, n_{\mathrm{GS}}} \\
\vdots & \ddots & \vdots & \ddots & \vdots \\
p_{g, i, 1} & \cdots & p_{g, i, j} & \cdots & p_{g, i, n_{\mathrm{GS}}} \\
\vdots & \ddots & \vdots & \ddots & \vdots \\
p_{g, n_{\mathrm{GS}}, 1} & \cdots & p_{g, n_{\mathrm{GS}}, j} & \cdots & p_{g, n_{\mathrm{GS}}, n_{\mathrm{GS}}}
\end{array}\right] \\
& \forall g \in \Omega_{\mathrm{DG}}
\end{aligned}
$$

The probabilistic scenarios are generated through the MCM, according to the flowchart presented in Fig. 3, where the main blocks (labeled as A, B, and C) are explained below.

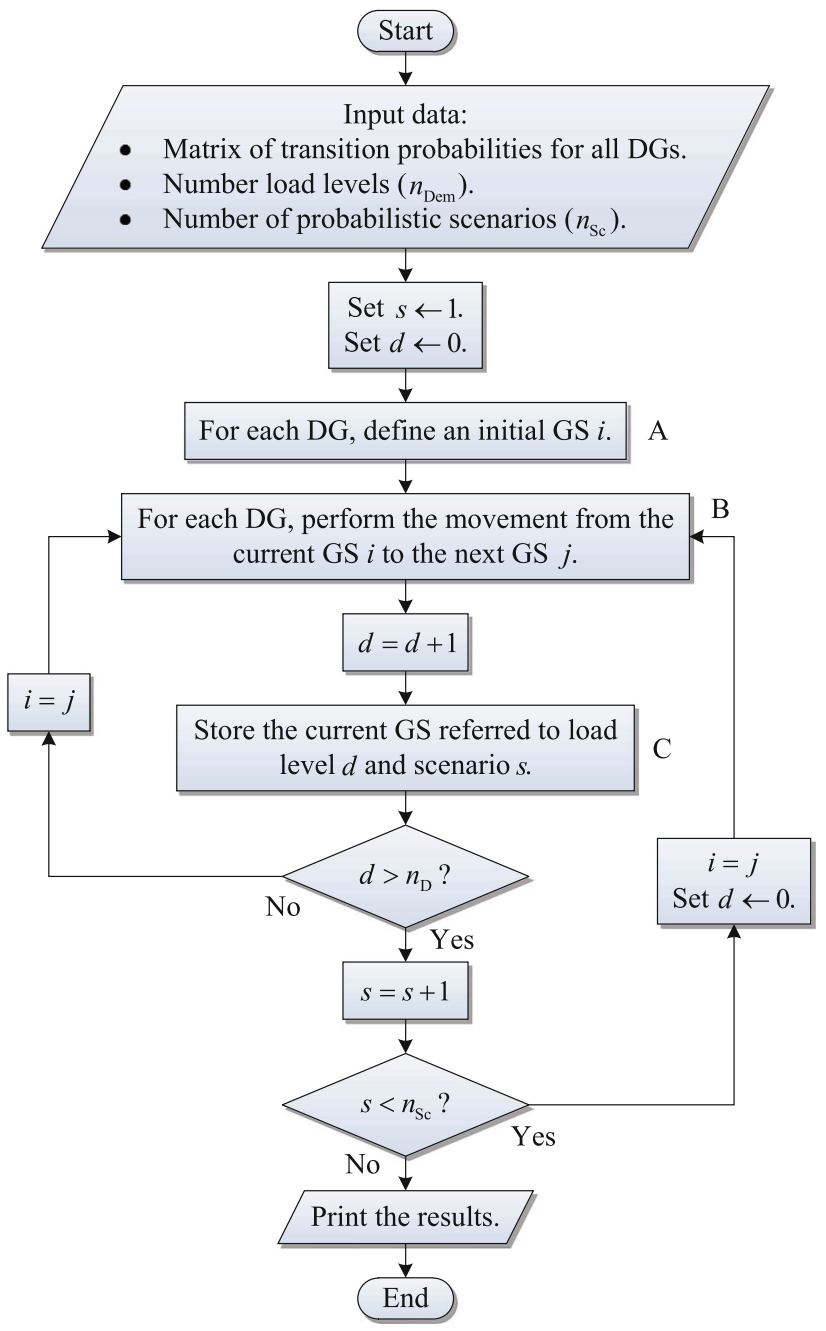

Fig. 3 Flowchart corresponding to the MCM algorithm (RuedaMedina and Padilha-Feltrin 2013)

1. Block A: For each DG $g$, the initial GS $i$ corresponds to the initial upper limit of active power generation in load level $d$, i.e., the initial value of $P_{\mathrm{DG}, g, d}^{\mathrm{Max}}$.

2. Block B: For each DG $g$, generate a uniform random number between 0 and 1 . Link these numbers to $p_{i, j}$, from the matrix $\widetilde{P}_{g}$ associated with the corresponding DG $g$, to move from the current GS $i$ to the next GS $j$, i.e., to define the value of $P_{\mathrm{DG}, g, d}^{\mathrm{Max}}$ for the next load level $d$, say $P_{\mathrm{DG}, g, d+1}^{\mathrm{Max}}$. 


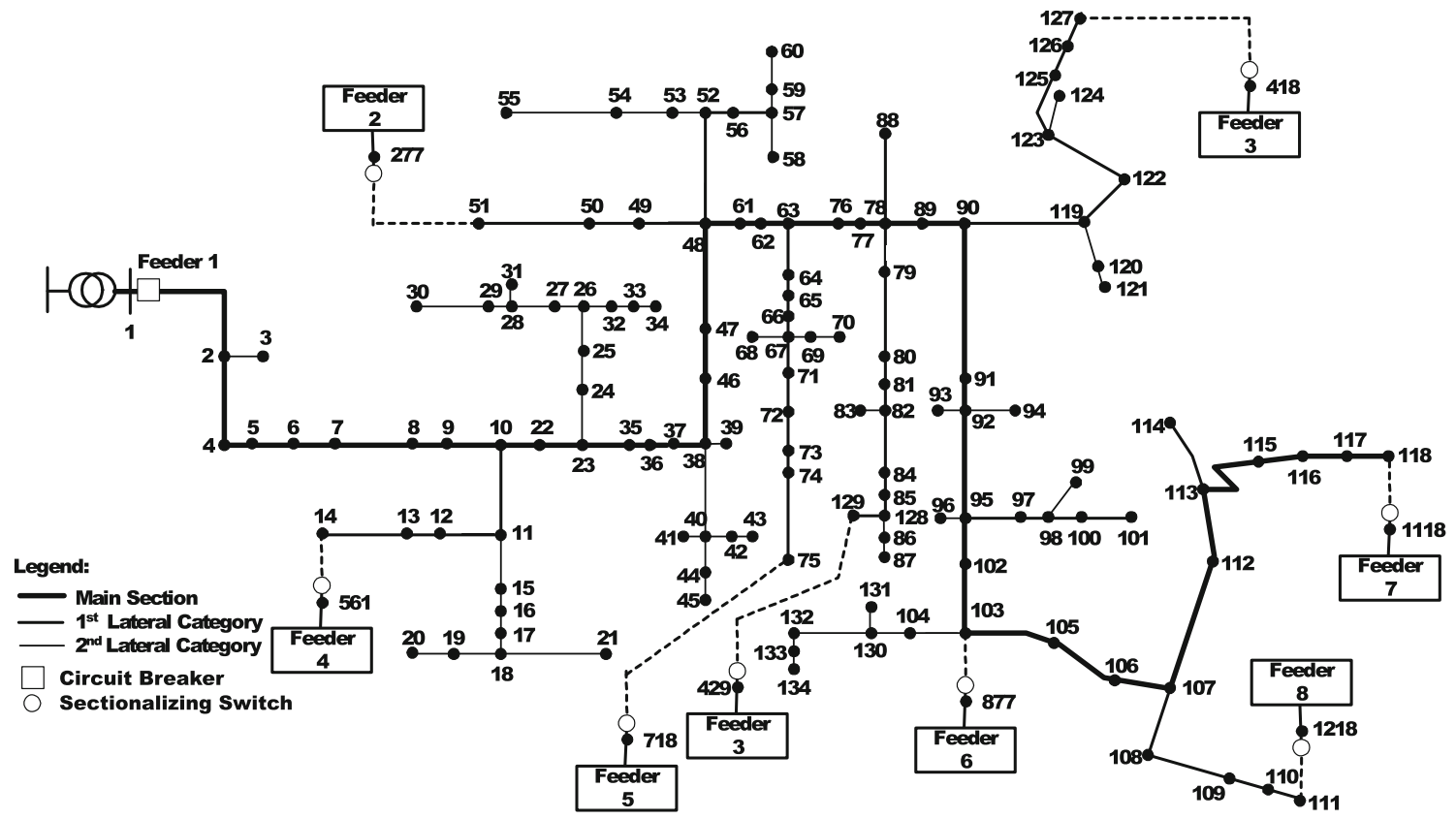

Fig. 4 Distribution feeder 135 buses, $13.8 \mathrm{kV}$

3. Block C: For each DG $g$, the value of $P_{\mathrm{DG}, g, d}^{\mathrm{Max}}$ is determined by the corresponding GS $j$ generated in Block B.

As presented above, the transition probabilities depend on collected statistical data (e.g., wind speed) and they form a matrix of transition probabilities $\widetilde{P}_{g}$. However, choosing the appropriate number of discretizations or GSs, that is, the size of $\widetilde{P}_{g}$, is very important for the efficiency of the forecast system. A very small number of GSs do not adequately represent the operation of the DGs, while a very large number of GSs could introduce large forecast errors. In this work, the best size of the matrix of transition probabilities is defined through a method proposed in Rueda-Medina et al. (2013).

\section{Results and Discussion}

The methodology proposed to solve the short-term EDSP problem was implemented in MATLAB, and it was tested in a 135-bus distribution feeder, which was adapted from [15], as shown in Fig. 4. The personal computer used for tests has a processor Intel Core $i 7 @ 2.00 \mathrm{GHz}$ with $16 \mathrm{~GB}$ of installed RAM. The modifications in the 135-bus distribution feeder consist of the inclusion of 8 neighboring feeders to simulate the relocation of loads for these feeders, as presented in Table 3.

The fault index $\lambda_{\mathrm{F}, s}$ used in the tests is 0.072 faults per year per kilometer. This value is associated with the conventional aerial structure. When changing conventional aerial struc-
Table 3 Reserve and load transfer capacities of the neighboring feeders of the 135-bus distribution feeder

\begin{tabular}{lll}
\hline Feeder number & $\begin{array}{l}\text { Reserve } \\
\text { capacity }(\mathrm{kW})\end{array}$ & $\begin{array}{l}\text { Load transfer } \\
\text { capacity }(\mathrm{kW})\end{array}$ \\
\hline 1 & 18.414 & 172.5 \\
2 & 11.910 & 618.4 \\
3 & 12.170 & 145.9 \\
4 & 12.750 & 640.1 \\
5 & 13.190 & 497.2 \\
6 & 21.603 & 179.4 \\
7 & 17.302 & 306.8 \\
8 & 21.800 & 251.7 \\
\hline
\end{tabular}

tures by compact or isolated structures, there is a reduction in $\lambda_{\mathrm{F}, s}$ to 0.022 and 0.011 faults per year per kilometer, respectively. This reduction is due to the fact that these compact and isolated structures have greater reliability than the conventional aerial ones. This information was achieved along an EDC from the south of Brazil.

Costs of cables mounted on conventional aerial, compact, and isolated structures used in tests are presented in Table 1.

Costs of fixed and switched CBs are shown in Table 4. These values are the same used in Park et al. (2009).

Costs of acquisition, installation, and maintenance of fuse links, reclosers, and directional relays are presented in Table 2; for all tests, costs of automatic switches are US\$ 200. All these cost values were obtained from planning sectors of Brazilian EDCs and from Peñuela and Mantovani (2013). 
Table 4 Costs of CBs

\begin{tabular}{lllllll}
\hline Reactive power (kVAr) & 300 & 600 & 900 & 1200 & 1500 \\
\hline Cost (US\$) & Fixed & 1904 & 1981 & 2519 & 2885 & 3106 \\
& Switched & 2865 & 2942 & 3673 & 3904 & 4212 \\
\hline
\end{tabular}

In all tests of this case, three DGs, each of $600 \mathrm{kVA}$, were considered to be allocated. Either the DGs as the CBs can be allocated in any bus of the distribution system.

In this work, the DG uncertainties are analyzed previously to the NSGA-II. Therefore, in order to define the matrix of transition probabilities $\widetilde{P_{g}}$, real historical data of wind speed (Swera, http://en.openei.org/wiki/SWERA/Data) corresponding to a period of 5 years, were used to construct the matrix of Table 5. In this matrix, a set of six discretized GSs were defined: The first one is associated with the minimum generation capacity (100 kVA), and the sixth one is associated with the maximum generation capacity $(600 \mathrm{kVA})$. The number of GSs was obtained according to the proposal presented in Rueda-Medina et al. (2013).

In all simulations, four load levels, i.e., $n_{\mathrm{D}}=4$, were considered. This number of load levels was chosen assuming that it is enough to adequately cover the range of load variations, and considering that this number is less than the number of GSs, which is enough to represent the intermittent characteristics of the DGs, as presented in Rueda-Medina et al. (2013). Periods of the day for these load levels, from 1 to 4 , respectively, are $00-07 \mathrm{~h}, 07-18 \mathrm{~h}, 18-21 \mathrm{~h}$, and $21 \mathrm{~h}$ to $00 \mathrm{~h}$. Each of these levels is related to a base load by a percentage value, as presented below:

- Load level 1 is $60 \%$ of the base load.

- Load levels 2 and 4 are $100 \%$ of the base load.

- Load level 3 is $130 \%$ of the base load.

Using the data of Table 5, considering 2,000 probabilistic scenarios, i.e., $n_{\mathrm{Sc}}=2,000$, the MCM algorithm proposed in Fig. 3 was executed. Among the 2,000 scenarios generated with this algorithm, the scenario with the greatest availability of energy that must be injected into the network, shown in Table 6, was chosen to be used in all simulations of the NSGA-II. Therefore, information of Table 6 corresponds to the generation capacity (in $\mathrm{kVA}$ ) of the DGs for each load level.

As a result of applying the proposed solution methodology, the non-dominated solutions presented in Table 7 were obtained. In this table, the results for each non-dominated solution, corresponding to technical losses and investment costs, are presented. Technical losses costs are related to the Joule effect in cables and non-supplied energy, while investment costs are related to costs of cables/structures, $\mathrm{CBs}$, and protection equipment. The computational time for
Table 5 Matrix of transition probabilities (in \%)

\begin{tabular}{lllllll}
\hline To GS From GS & 1 & 2 & 3 & 4 & 5 & 6 \\
\hline 1 & 0.658 & 0.167 & 0.087 & 0.046 & 0.028 & 0.012 \\
2 & 0.268 & 0.433 & 0.137 & 0.081 & 0.061 & 0.020 \\
3 & 0.160 & 0.305 & 0.317 & 0.119 & 0.053 & 0.045 \\
4 & 0.220 & 0.231 & 0.297 & 0.154 & 0.055 & 0.044 \\
5 & 0.064 & 0.106 & 0.128 & 0.213 & 0.227 & 0.213 \\
6 & 0.045 & 0.143 & 0.156 & 0.182 & 0.227 & 0.247 \\
\hline
\end{tabular}

Table 6 Apparent power (maximum generation capacity), in $\mathrm{kVA}$, of the DGs for each load level

\begin{tabular}{lllll}
\hline DG & \multicolumn{4}{l}{ Demand level } \\
\cline { 2 - 5 } & 1 & 2 & 3 & 4 \\
\hline 1 & 600 & 600 & 200 & 600 \\
2 & 500 & 500 & 500 & 300 \\
3 & 500 & 600 & 300 & 600 \\
\hline
\end{tabular}

obtaining the results presented in this table was $4 \mathrm{~h} 13 \mathrm{~min}$ and $20 \mathrm{~s}$.

The results presented in Table 7 are depicted in Fig. 5. As shown in this table and figure, there is a reduction of losses when the system investment is increased. This is due to the fact that, as more investment is applied to the system, more robust and reliable network becomes, reducing losses and possible failures.

In order to obtain the best results, the NSGA-II requires making adjustments in the genetic parameters (number of the population's individuals, mutation rate for the genes, crossover rate, number of individuals by tournament, and stopping criterion) and in the initial population; these adjustments were performed through a trial-and-error process. The best values for these parameters, through which the results presented in Table 7 and Fig. 5 were obtained, are: number of the population's individuals equal to 100 ; mutation rate for the genes (individuals of population set) equal to 0.05 ; crossover rate equal to 0.9 ; number of individuals by tournament equal to 2; and stopping criterion equal to 100 generations. As an example of the results obtained through the trial-and-error process commented before, some non-dominated fronts are presented in Fig. 6, for several seeds used in the pseudorandom initial population generation, and in Fig. 7, for different sets of genetic parameters.

In Fig. 6, note that Front-Seed 1 could be chosen as a good-quality front; however, the non-dominated front of Fig. 5 was chosen as the "best front" because most of the solutions of this front are better than solutions of FrontSeed 1 . Note also that there were some seeds that led to very bad-quality fronts in terms of diversity, such as Front-Seed 2, Front-Seed 3, and Front-Seed 5, since they provided only 2,4 , and 4 solutions, respectively. 
Table 7 Technical losses and investment costs of solutions of the non-dominated front

\begin{tabular}{|c|c|c|c|c|c|}
\hline \multirow[t]{2}{*}{ Solution } & \multicolumn{2}{|c|}{ Technical losses costs (US\$) } & \multicolumn{3}{|c|}{ Investment costs (US\$) } \\
\hline & Joule effect & Non-supplied energy & Cables/structures & CBs & Protection equipment \\
\hline $1(\mathrm{~A})$ & $629,764.20$ & $16,660.77$ & $75,791.24$ & 0.00 & $7,500.00$ \\
\hline 2 & $607,207.12$ & $17,558.71$ & $76,743.41$ & 0.00 & $14,800.00$ \\
\hline 3 & $585,365.71$ & $12,080.94$ & $80,588.85$ & $7,711.54$ & $9,000.00$ \\
\hline 4 & $558,502.32$ & $15,428.90$ & $78,348.93$ & $13,442.31$ & $7,800.00$ \\
\hline 5 & $511,683.75$ & $17,319.08$ & $81,036.34$ & $13,442.31$ & $6,700.00$ \\
\hline 6 & $512,110.68$ & $12,679.13$ & $81,127.64$ & $13,442.31$ & $7,400.00$ \\
\hline $7(\mathrm{~B})$ & $485,244.78$ & $13,998.32$ & $82,926.28$ & $12,500.00$ & $8,100.00$ \\
\hline 8 & $467,495.69$ & $18,260.96$ & $82,023.63$ & $20,211.54$ & $7,500.00$ \\
\hline 9 & $463,966.22$ & $12,737.39$ & $82,133.50$ & $21,153.85$ & $7,400.00$ \\
\hline 10 & $465,841.57$ & $10,097.92$ & $82,228.40$ & $21,153.85$ & $9,200.00$ \\
\hline 11 & $458,685.14$ & $15,891.16$ & $83,550.89$ & $21,153.85$ & $14,200.00$ \\
\hline 12 & $452,334.29$ & $11,784.04$ & $83,776.98$ & $21,153.85$ & $14,400.00$ \\
\hline $13(\mathrm{C})$ & $452,887.54$ & $10,971.71$ & $91,516.21$ & $21,153.85$ & $17,900.00$ \\
\hline
\end{tabular}

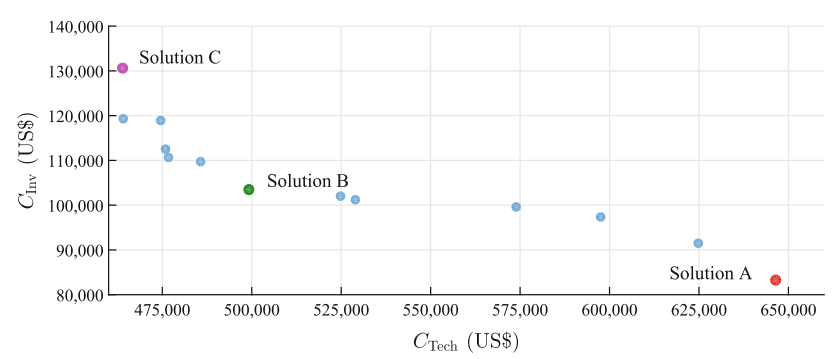

Fig. 5 Non-dominated solutions

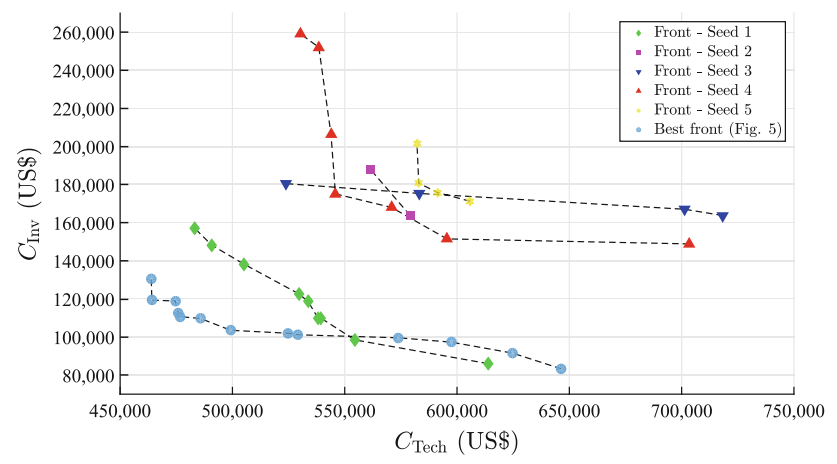

Fig. 6 Non-dominated fronts with different initial populations, i.e., with several seeds used in the pseudorandom initial population generation

In Fig. 7, note that Front-Parameters set 1, FrontParameters set 2, and Front-Parameters set 3 represent bad-quality solutions in terms of diversity, since they provided only 7,5 , and 5 solutions, respectively, against 13 good-quality solutions of the non-dominated front of Fig. 5.

The equipment installed in the network, corresponding to each solution of the non-dominated front presented in Fig. 5, is detailed in Table 8 .

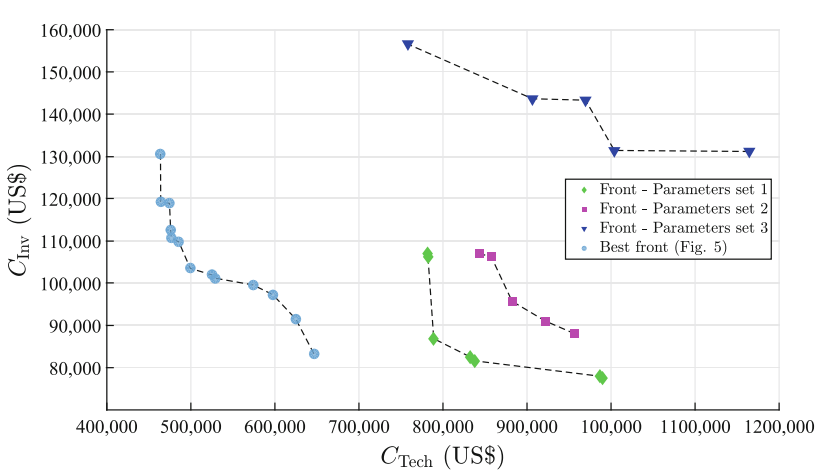

Fig. 7 Non-dominated fronts with different sets of genetic parameters (number of the population's individuals, mutation rate for the genes, crossover rate, number of individuals by tournament, and stopping criterion)

In Table 8, note the diversity of elements composing the solutions. In the first two solutions, with smaller investment costs, the $\mathrm{CBs}$ are not required to be installed; the allocation of $\mathrm{CBs}$ in solutions 3 to 13 interferes in the two objective functions considered in this work, increasing the investment costs and reducing the technical losses, as it could be confirmed in Table 7 , since these capacitors are allocated in regions that concentrate higher densities of loads. Solution 2 is the only one with a recloser, and eight solutions (a little over half) require only one directional relay. The number of fuse links and switches remains almost invariant for all solutions, except for Solution 13 (or C), which requires almost twice fuse links. Solution $\mathrm{C}$ has the biggest number of elements to be installed causing, thereby, the smallest losses costs, US $\$ 463,859.25$, and the biggest investment costs, US\$130,570.06. When comparing Solutions C and 1 (or 
Table 8 Equipment installed in the network for each non-dominated solution

\begin{tabular}{llllll}
\hline Solution & CBs & Fuse links & Switches & Reclosers & Directional relays \\
\hline $1($ A) & 0 & 11 & 7 & 0 & 1 \\
2 & 0 & 12 & 10 & 1 & 1 \\
3 & 1 & 14 & 7 & 0 & 1 \\
4 & 2 & 12 & 6 & 0 & 1 \\
5 & 2 & 10 & 7 & 0 & 1 \\
6 & 2 & 11 & 8 & 0 & 1 \\
$7($ B $)$ & 2 & 13 & 9 & 0 & 1 \\
8 & 3 & 13 & 6 & 0 & 3 \\
9 & 3 & 11 & 8 & 0 & 3 \\
10 & 3 & 14 & 8 & 0 & 3 \\
11 & 3 & 12 & 7 & 0 & 3 \\
12 & 3 & 12 & 8 & 0 & 1 \\
$13(\mathrm{C})$ & 3 & 19 & 9 & 0 & \\
\hline
\end{tabular}

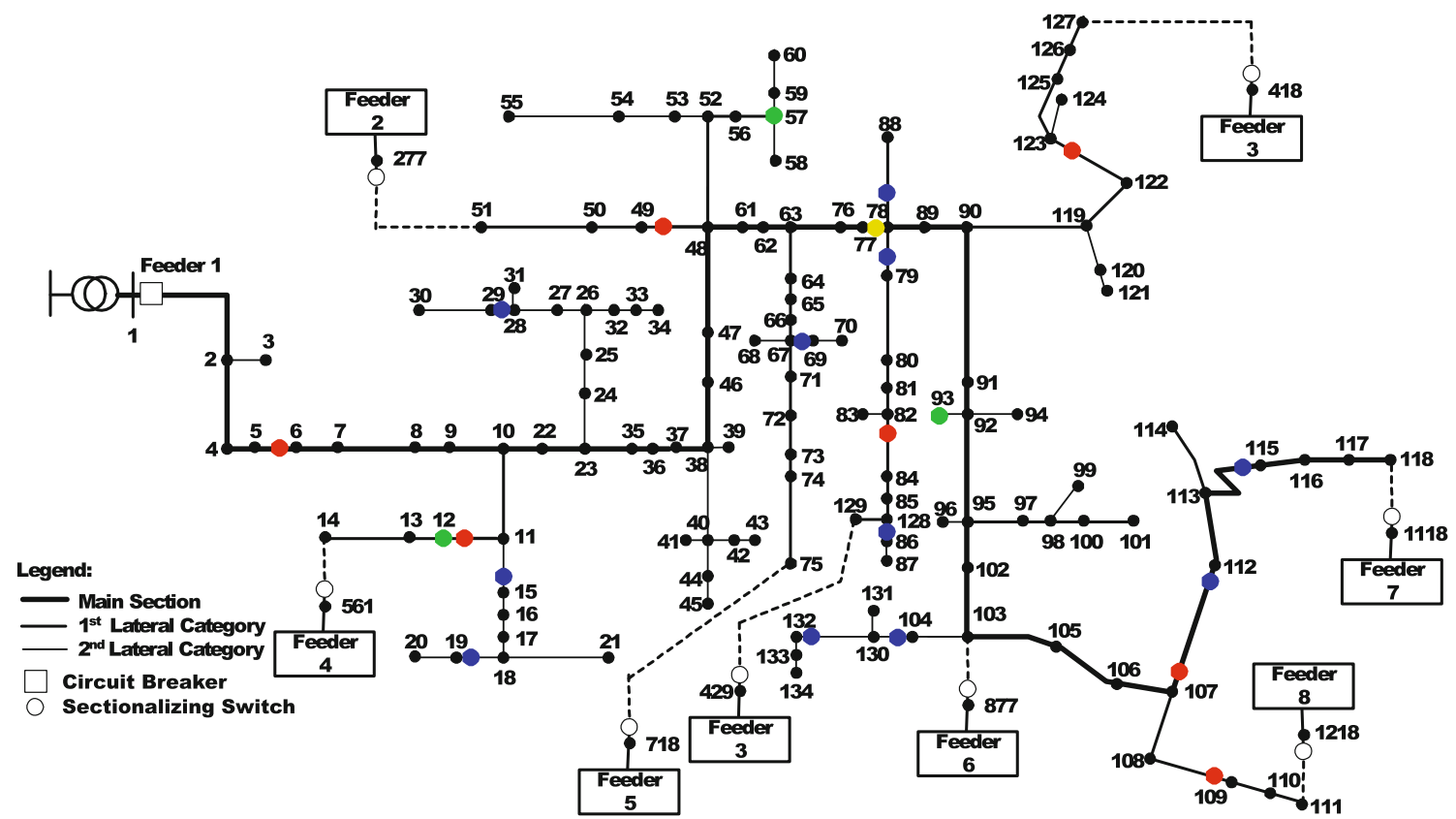

Fig. 8 Elements allocated in Solution A

A), Solution $\mathrm{C}$ has an increase of $36.21 \%$ in investment and a reduction of $28.24 \%$ in losses.

In Figs. 8, 9, and 10, the elements allocated according to Solutions A, B, and C (in Table 8), respectively, are shown. In these figures, DGs, CBs, fuse links, switches, and directional relays are represented by green, purple, blue, red, and yellow circles, respectively.

In order to make comparisons, tests without DGs were performed. After applying the proposed solution methodology, but neglecting the DGs, the non-dominated solutions presented in Table 9 were obtained. Similar to Table 7, these results correspond to technical losses, which are related to the
Joule effect in cables and non-supplied energy, and investment costs, which are related to costs of cables/structures, $\mathrm{CBs}$, and protection equipment.

In Fig. 11, the results presented in Tables 7 and 9, corresponding to the cases with and without DGs, respectively, are depicted. Note the significant costs reduction associated with losses when the DGs are considered to be installed in the network. This reduction corresponds to 175,028.02US\$, which means a reduction percentage of $21.31 \%$, when comparing the extreme Solutions $\mathrm{A}$ and $\mathrm{A}^{\prime}$ in Fig. 11, and $176,360.79$ US $\$$, which means a reduction percentage of $25.55 \%$, when comparing the extreme Solutions $C$ and $\mathrm{C}^{\prime}$ in 


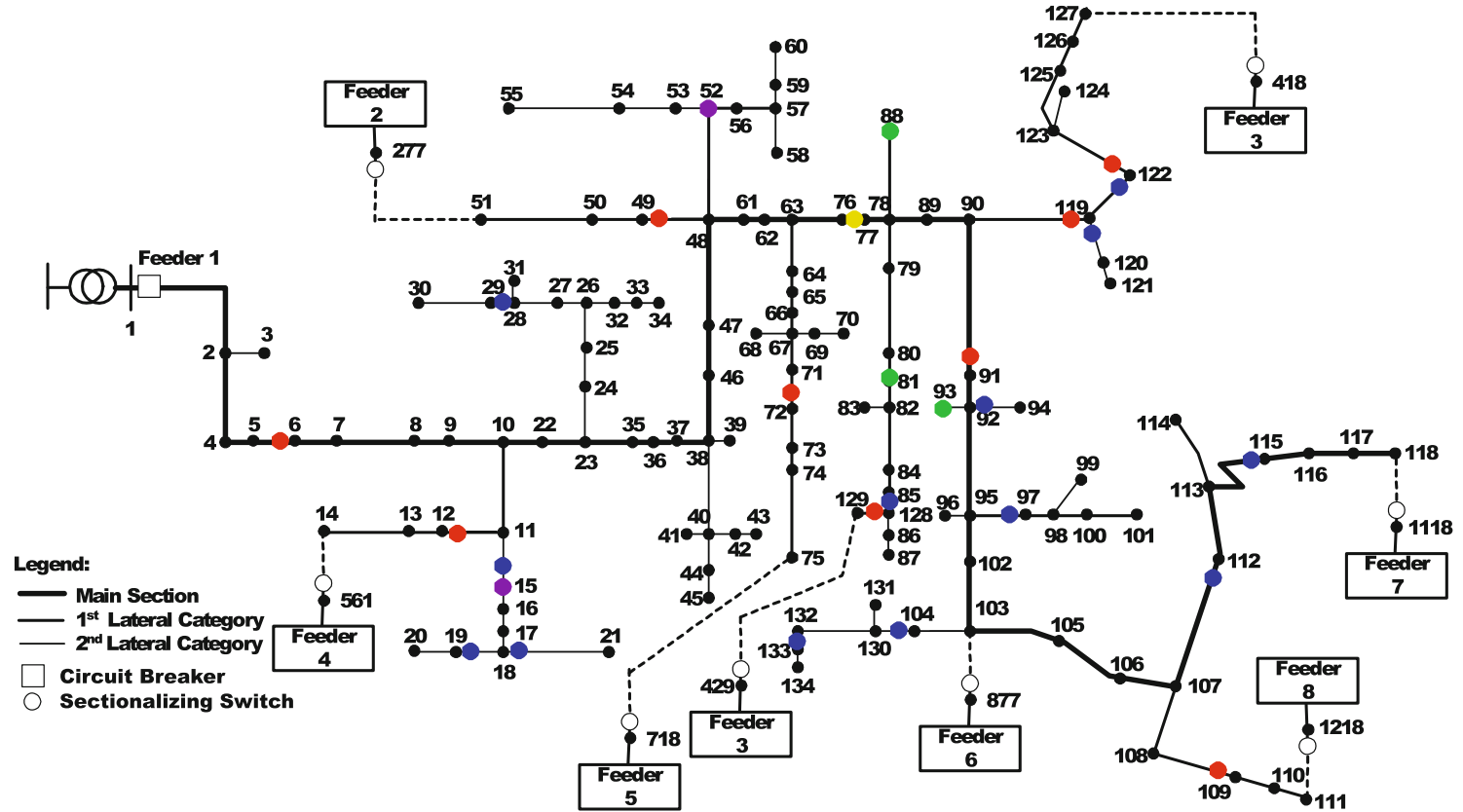

Fig. 9 Elements allocated in Solution B

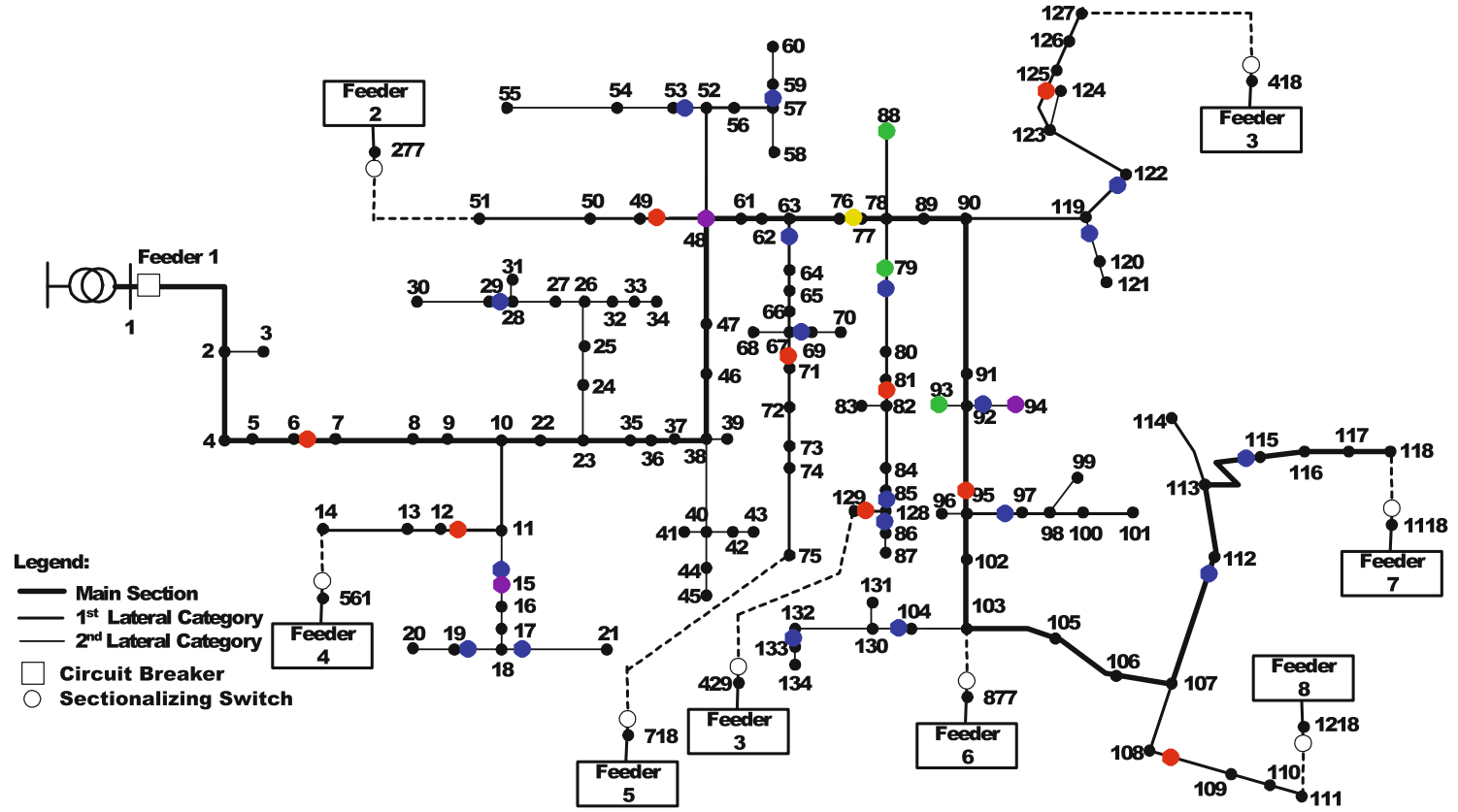

Fig. 10 Elements allocated in Solution C

the same figure. Note also that these costs reduction in losses is achieved with additional investments of 12,794.13US\$ for extreme Solutions A and $A^{\prime}$ and 20,956.21 US\$ for extreme Solutions $\mathrm{C}$ and $\mathrm{C}^{\prime}$. Although the DGs installation costs are not considered, since these costs are constant values which do not affect the optimization process, it is important to remark that the DGs reward for active power provided to the system is extended to more than the planning horizon of 5 years assumed in test developed in this work. Thus, for example, considering an active power price of $12 \$$ per $\mathrm{kW} / \mathrm{h}$ and a investment return rate of $8 \%$ for the same period of the planning horizon, the DGs rewards for each non-dominated solution are those presented in Table 10, which were updated to future values using expression (18).

$F V=P V(1+\alpha)^{n_{\mathrm{Yr}}}$ 
Table 9 Technical losses and investment costs of solutions of the non-dominated front (without DGs)

\begin{tabular}{|c|c|c|c|c|c|}
\hline \multirow[t]{2}{*}{ Solution } & \multicolumn{2}{|c|}{ Technical losses costs (US\$) } & \multicolumn{3}{|c|}{ Investment costs (US\$) } \\
\hline & Joule effect & Non-supplied energy & Cables/structures & $\mathrm{CBs}$ & Protection equipment \\
\hline 1 & $798,370.23$ & $23,082.76$ & $61,697.11$ & 0.00 & $8,800.00$ \\
\hline 2 & $792,067.97$ & $23,313.02$ & $64,321.31$ & 0.00 & $8,100.00$ \\
\hline 3 & $792,123.87$ & $21,018.13$ & $63,799.31$ & $2,865.38$ & $9,000.00$ \\
\hline 4 & $743,174.56$ & $25,032.52$ & $62,917.33$ & $4,769.23$ & $8,900.00$ \\
\hline 5 & $723,028.59$ & $29,478.78$ & $60,833.84$ & $7,711.54$ & $8,700.00$ \\
\hline 6 & $708,599.81$ & $15,659.05$ & $64,540.12$ & $4,846.15$ & $9,900.00$ \\
\hline 7 & $696,939.91$ & $18,633.26$ & $62,914.49$ & $9,615.38$ & $11,100.00$ \\
\hline 8 & $689,020.13$ & $18,143.96$ & $62,914.49$ & $9,615.38$ & $11,300.00$ \\
\hline 9 & $679,112.26$ & $23,124.35$ & $62,917.33$ & $12,480.77$ & $8,600.00$ \\
\hline 10 & $676,249.96$ & $12,833.85$ & $62,917.33$ & $13,519.23$ & $11,700.00$ \\
\hline 11 & $659,511.94$ & $22,313.65$ & $62,917.33$ & $18,288.46$ & $9,100.00$ \\
\hline 12 & $659,583.37$ & $18,597.71$ & $63,549.20$ & $18,288.46$ & $11,800.00$ \\
\hline 13 & $635,783.89$ & $24,399.93$ & $65,533.97$ & $18,288.46$ & $11,500.00$ \\
\hline 14 & $630,881.11$ & $26,327.99$ & $64,895.67$ & $25,057.69$ & $8,700.00$ \\
\hline 15 & $630,881.11$ & $23,587.76$ & $64,895.67$ & $25,057.69$ & $8,900.00$ \\
\hline 16 & $630,881.11$ & $19,454.82$ & $64,895.67$ & $25,057.69$ & $10,200.00$ \\
\hline 17 & $630,881.11$ & $19,387.17$ & $64,895.67$ & $25,057.69$ & $11,400.00$ \\
\hline 18 & $629,544.92$ & $13,208.18$ & $66,165.84$ & $29,038.46$ & $11,500.00$ \\
\hline 19 & $629,474.48$ & $12,674.52$ & $65,533.97$ & $29,038.46$ & $12,200.00$ \\
\hline 20 & $629,214.29$ & $11,005.75$ & $68,575.39$ & $29,038.46$ & $12,000.00$ \\
\hline
\end{tabular}

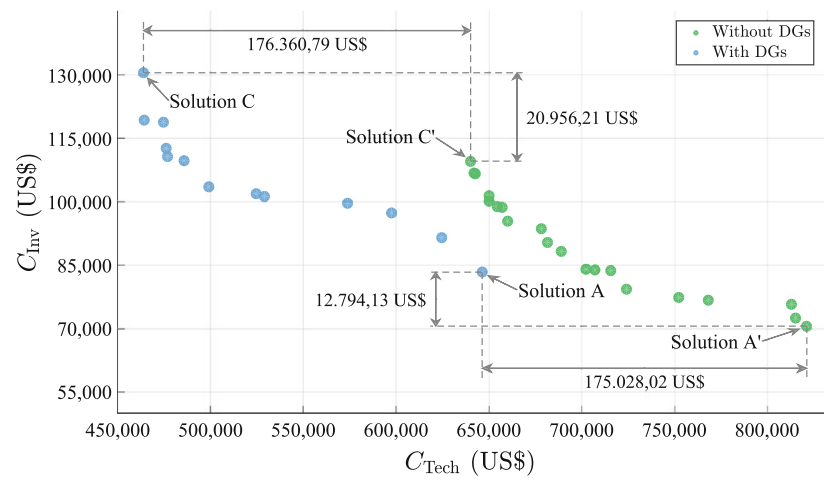

Fig. 11 Non-dominated solutions (comparison without and with DGs)

\section{Conclusions}

The methodology proposed in this work for the short-term distribution systems planning provides a set of optimized solutions, which enables the distribution network planner, along with investors of distributed generation, to make investment decisions that address technical and economic interests of these two agents.

Another important feature of the work presented in this paper is the consideration of the uncertainties present in the DGs based on renewable energy sources. Through a forecast-
Table 10 DGs reward in 5 years

\begin{tabular}{lllll}
\hline Solution & $\mathrm{DG}_{1}(\mathrm{US} \$)$ & $\mathrm{DG}_{2}$ (US\$) & $\mathrm{DG}_{3}(\mathrm{US} \$)$ & Total $(\mathrm{US} \$)$ \\
\hline 1 (A) & $3,194,294.86$ & $2,844,459.00$ & $3,081,978.44$ & $9,120,732.30$ \\
2 & $3,194,294.86$ & $2,844,459.00$ & $3,037,585.62$ & $9,076,339.48$ \\
3 & $3,194,294.86$ & $2,810,368.77$ & $3,024,038.91$ & $9,028,702.54$ \\
4 & $3,194,294.86$ & $2,810,368.77$ & $3,024,038.91$ & $9,028,702.54$ \\
5 & $3,134,097.55$ & $2,810,368.77$ & $3,113,638.79$ & $9,058,105.11$ \\
6 & $3,134,097.55$ & $2,810,368.77$ & $3,024,038.91$ & $8,968,505.23$ \\
7 (B) & $3,134,097.55$ & $2,810,368.77$ & $3,127,185.50$ & $9,071,651.82$ \\
8 & $3,134,097.55$ & $2,810,368.77$ & $3,127,185.50$ & $9,071,651.82$ \\
9 & $3,134,097.55$ & $2,810,368.77$ & $3,127,185.50$ & $9,071,651.82$ \\
10 & $3,134,097.55$ & $2,820,494.13$ & $3,037,585.62$ & $8,992,177.29$ \\
11 & $3,134,097.55$ & $2,810,368.77$ & $3,046,231.47$ & $8,990,697.79$ \\
12 & $3,134,097.55$ & $2,844,459.00$ & $3,068,431.73$ & $9,046,988.29$ \\
13 (C) & $3,134,097.55$ & $2,844,459.00$ & $3,081,978.44$ & $9,060,534.99$ \\
\hline
\end{tabular}

ing methodology, which combines the MCM and MkvM, the amount of active power to be injected into the network by the DGs is defined in a pre-processing stage (before applying the NSGA-II).

The obtained results show the efficiency of the proposed methodology, helping to maintain the quality of the energy supply and the reliability of the distribution network, while reducing technical losses with a low investment cost. 
Acknowledgements This work was supported partially by Fundação de Amparo à Pesquisa do Estado de São Paulo (FAPESP), Grant Numbers 2012/15210-8 and 2013/23590-8, and Conselho Nacional de Desenvolvimento Científico e Tecnológico (CNPq), Grand Number 305371/2012-6.

\section{References}

Baran, M., \& Wu, F. F. (1989). Optimal sizing of capacitors placed on a radial distribution system. IEEE Transactions on Power Delivery, 4(1), 735-743.

Chiang, H. D., Wang, J. C., Cockings, O., \& Shin, H. D. (1990). Optimal capacitor placements in distribution systems-II: Solution algorithms and numerical results. IEEE Transactions on Power Delivery, 5(2), 643-649.

Deb, K. (2009). Multi-objective optimization using evolutionary algorithms (1st ed.). New York, NY: Wiley.

Deb, K., Pratap, A., Agarwal, S., \& Meyarivan, T. (2002). A fast and elitist multiobjective genetic algorithm: NSGA-II. IEEE Transactions on Evolutionary Computation, 6(2), 182-197.

El-kathan, W., Hegazy, Y. G., \& Salama, M. M. A. (2005). An integrated distributed generation optimization model for distribution system planning. IEEE Transactions on Power Systems, 20(2), $1158-1165$.

Gallego, R. A., Monticelli, A. J., \& Romero, R. (2001). Optimal capacitor placement in radial distribution networks. IEEE Transactions on Power Systems, 16(4), 630-637.

Gonen, T. (1985). Electric power distribution system engineering (3rd ed.). New York, NY: Mcgraw-Hill College.

LaPSEE. Laboratory of Electrical Power System Planning. Practical 135 bus feeder data. On line: http://www.feis.unesp.br/\#!/ departamentos/engenharia-eletrica/pesquisas-e-projetos/lapsee/ english/downloads/testing-systems/, Department of Electrical Engineering of Ilha Solteira, Universidade Estadual Paulista UNESP.

Mandal, S., \& Pahwa, A. (2002). Optimal selection of conductors for distribution feeders. IEEE Transactions on Power Systems, 17(1), 192-197.

Mendoza, F., Requena, D., Bemal-Agustin, J., \& Dominguez-Navarro, J. (2006). Optimal conductor size selection in radial power distribution systems using evolutionary strategies. IEEE Transmission and Distribution Conference and Exposition: Latin America, 2006, $1-5$.
Padilha-Feltrin, A., Quijano-Rodezno, D. A., \& Mantovani, J. R. S. (2015). Volt-VAR multiobjective optimization to peak-load relief and energy efficiency in distribution networks. IEEE Transactions on Power Delivery, 30(2), 618-626.

Park, J. Y., Sohn, J. M., \& Park, J. K. (2009). Optimal capacitor allocation in a distribution system considering operation costs. IEEE Transactions on Power Systems, 24(1), 462-468.

Peñuela, C. A., \& Mantovani, J. R. S. (2013). Improving the grid operation and reliability cost of distribution systems with dispersed generation. IEEE Transactions on Power Systems, 28(3), 24852496.

Pereira, B. R, Jr., Cossi, A. M., \& Mantovani, J. R. S. (2013). Multiobjective short-term planning of electric power distribution systems using NSGA-II. Elsevier Journal of Control, Automation and Electrical Systems, 24(3), 286-299.

Rueda-Medina, A. C., Padilha-Feltrin, A. \& Mantovani, J. R. S. (2013). Efficient forecasting system for distributed generators with uncertainties in the primary energy source. In 22th international conference and exhibition on electricity distribution CIRED, 2013, (pp. 1-4).

Rueda-Medina, A. C., \& Padilha-Feltrin, A. (2013). Distributed generators as providers of reactive power support-a market approach. IEEE Transactions on Power Systems, 28(1), 490-502.

Shirmohammadi, D., Hong, H. W., \& Luo, G. X. (1988). Compensationbased power flow method for weakly meshed distribution and transmission networks. IEEE Transactions on Power Systems, 3(2), 753-762.

Souza, B. A., Alves, H. N., \& Ferreira, H. A. (2004). Microgenetic algorithms and fuzzy logic applied to the optimal placement of capacitor banks in distribution networks. IEEE Transactions on Power Systems, 19(2), 942-947.

Sundharajan, S., \& Pahwa, A. (1994). Optimal selection of capacitors for radial systems using a genetic algorithm. IEEE Transactions on Power Delivery, 9(3), 1499-1505.

Swera. Solar and Wind Energy Resource Assessment. Brazil wind data. On line: http://en.openei.org/wiki/SWERA/Data.

Tan, W., Hassan, M. Y., Majid, M. S., \& Rahman, H. A. (2013). Optimal distributed renewable generation planning: A review of different approaches. Renewable and Sustainable Energy Reviews, 18, 626645.

Vahidet, M., Manouchehr, N., Hossein, S. D., \& Jamaleddin, A. (2009). Combination of optimal conductor selection and capacitor placement in radial distribution systems for maximum loss reduction. In IEEE International Conference on Industrial Technology, 2009, (pp. 1-5). 